PROCEEDINGS OF THE

AMERICAN MATHEMATICAL SOCIETY

Volume 138, Number 5, May 2010, Pages 1667-1672

S 0002-9939(09)10228-9

Article electronically published on December 18, 2009

\title{
A CAUCHY-RIEMANN EQUATION FOR GENERALIZED ANALYTIC FUNCTIONS
}

\author{
JOHN WERMER
}

(Communicated by Franc Forstneric)

\begin{abstract}
We denote by $T^{2}$ the torus: $z=\exp i \theta, w=\exp i \phi$, and we fix a positive irrational number $\alpha$. $A_{\alpha}$ denotes the space of continuous functions $f$ on $T^{2}$ whose Fourier coefficient sequence is supported by the lattice half-plane $n+m \alpha \geq 0$. R. Arens and I. Singer introduced and studied the space $A_{\alpha}$, and it turned out to be an interesting generalization of the disk algebra. Here we construct a differential operator $X_{\Sigma}$ on a certain 3-manifold $\Sigma_{0}$ such that $X_{\Sigma}$ characterizes $A_{\alpha}$ in a manner analogous to the characterization of the disk algebra by the Cauchy-Riemann equation in the disk.
\end{abstract}

\section{INTRODUCTION}

Let $\Gamma$ be the unit circle. The disk algebra $A$ on $\Gamma$ is the space of all continuous functions $f$ on $\Gamma$ such that the Fourier expansion of $f$ is:

$$
\sum_{n=0}^{\infty} c_{n} \exp (i n \theta)
$$

i.e., the Fourier coefficient sequence of $f$ is supported on the semi-group $n \geq 0$ of $Z$.

In 1, R. Arens and I.M. Singer studied the following generalization of the disk algebra: we replace $\Gamma$ by the 2-torus $T^{2}$ and fix a positive irrational number $\alpha$. The dual group of $T^{2}$ is $Z \oplus Z$. We replace the semi-group of nonnegative integers by the semi-group of all pairs of integers $(n, m)$ with $n+m \alpha \geq 0$. We define the algebra $A_{\alpha}$ as the space of continuous functions on $T^{2}$ with Fourier expansion on the torus given by

$$
\sum_{n+m \alpha \geq 0} c_{n m} \exp (i n \theta) \exp (i m \phi) .
$$

$A_{\alpha}$ is called a space of Generalized Analytic Functions. In [4, H. Helson and D. Lowdenslager made a detailed study of $A_{\alpha}$ and showed that many basic results of analytic function theory on the unit disk extend from $A$ to $A_{\alpha}$.

An alternative description to the disk algebra is the following: $A$ consists of those functions $f$ continuous on $\Gamma$ which admit a continuous extension to the closed disk $\Delta$, again denoted by $f$, such that $f$ is smooth on the interior of $\Delta$ and there it

Received by the editors May 8, 2009.

2000 Mathematics Subject Classification. Primary 32-XX.

(C)2009 American Mathematical Society Reverts to public domain 28 years from publication 1667 
satisfies the equation

$$
\frac{\delta}{\delta \bar{z}}(f)=0 .
$$

In [1] the disk $\Delta$ is replaced by the maximal ideal space $\Pi$ of the Banach algebra $A_{\alpha}$, taken in the Gelfand topology. It is shown in [1] that $\Pi$ has a natural identification with the following compact subset of $C^{2}$ :

The set of all points $(z, w)$ in $C^{2}$ such that $|w|=|z|^{\alpha}$ and $|z| \leq 1$.

We denote this subset of $C^{2}$ by $\Sigma$. In this identification, $T^{2}$ turns into the set of all points $(z, w) \in C^{2}$ such that $|z|=|w|=1$.

Our purpose is to give an equation analogous to (1) on the space $\Sigma \backslash\left(T^{2} \cup 0\right)$, which characterizes $A_{\alpha}$. To this end we define the differential operator $\mathrm{X}$ on $C^{2}$ by $X=\bar{z} \frac{\delta}{\delta \bar{z}}+\alpha \bar{w} \frac{\delta}{\delta \bar{w}}$. As is shown below, $X$ restricts to a well-defined differential operator on the smooth manifold: $\Sigma \backslash T^{2} \cup\{0\}$, which we denote by $\Sigma_{0}$.

Theorem 1.1. A function $f \in C\left(T^{2}\right)$ lies in $A_{\alpha}$ if and only if $f$ admits a continuous extension (denoted $F$ ) to $\Sigma$ such that $X F=0$, in the sense of distributions, on $\Sigma \backslash\left(T^{2} \cup 0\right)$.

Theorem 1.2. Given a point $\left(z_{0}, w_{0}\right) \in \Sigma \backslash\left(T^{2} \cup 0\right)$, there exists some function $f$ in $A_{\alpha}$ such that the extension $F$ is not differentiable on $\Sigma$ at that point.

\section{Proof of Theorem 1.1}

We put $\Sigma_{0}=\Sigma \backslash T^{2} \cup(0,0)$.

Let $\phi$ be the function on $C^{2} \backslash z=0$ given by $\phi(z, w)=w \bar{w}-z^{\alpha} \bar{z}^{\alpha}$.

$\Sigma$ has the equation: $\phi(z, w)=0$. We write $D_{\bar{z}}$ for the derivative with respect to $\bar{z}$ and similarly for $w$. $X(\phi)=\alpha \phi$, by direct calculation. So $X(\phi)=0$ on $\Sigma$.

Since $\Sigma$ is given by the equation $\phi=0$, it follows that the operator $X$ is welldefined on $C^{\infty}(\Sigma)$. We denote this operator, which acts on functions defined on $\Sigma$, by $X_{\Sigma}$. We wish to express $X_{\Sigma}$ in local coordinates on $\Sigma$. Fix a point $\left(z_{0}, w_{0}\right)$ on $\Sigma$. Then $w_{0}=z_{0}^{\alpha} \exp \left(i \theta_{0}\right)$ for some $z_{0}, \theta_{0}$ with $\left|z_{0}\right|<1,0 \leq \theta_{0} \leq 2 \pi$. We define a neighborhood $U$ of $\left(z_{0}, w_{0}\right)$ on $\Sigma$ by:

$$
U=\text { the set of }\left(t, \exp (i \theta) t^{\alpha}\right),\left|t-z_{0}\right|<\delta,\left|\theta-\theta_{0}\right|<\delta .
$$

We fix a single-valued branch of $t^{\alpha}$ near $t=z_{0}$.

We use $t, \bar{t}, \theta$ as local coordinates in $U$. Further, we denote the operator $\frac{\delta}{\delta t}$ by $D_{\bar{t}}$.

Claim 1. $\bar{t} D_{\bar{t}}=X_{\Sigma}$ as a differential operator on $U$. Hence for a continuous function $f$ on $U, t D_{\bar{t}} f=X_{\Sigma} f$, as a distribution on $U$.

Proof of Claim 1. We apply both sides to the functions $t, \bar{t}, \exp (i \theta), \exp (-i \theta)$. We note that $\bar{t}$ is the restriction of $\bar{z}$ to $\Sigma$. Since $X=\bar{z} D_{\bar{z}}+\alpha \bar{w} D_{\bar{w}}, X_{\Sigma}(\bar{t})=\bar{t}$ on $U$. Next, $t$ is the restriction of $z$ to $\Sigma$. So $X_{\Sigma}(t)=0$. Next, $X_{\Sigma}(\exp (i \theta))=X\left(\frac{w}{z^{\alpha}}\right)=$ 0 . Similarly, $X_{\Sigma}(\exp (-i \theta))=0$. On the other hand, $\bar{t} D_{\bar{t}}(\bar{t})=\bar{t}, \bar{t} D_{\bar{t}}(t)=0$, $\bar{t} D_{\bar{t}}(\exp (i \theta))=0, \bar{t} D_{\bar{t}}(\exp (-i \theta))=0$.

So $X_{\Sigma}$ and $\bar{t} D_{\bar{t}}$ agree on each of the functions $t, \bar{t}, \exp i \theta$ on $U$. Also, $0=$ $X_{\Sigma}(\exp i \theta)=i \exp i \theta X_{\Sigma}(\theta)$, so $X_{\Sigma}(\theta)=0$. Similarly, $\bar{t} D_{\bar{t}}(\theta)=0$. It follows that for all $G$ in $C^{\infty}(U)$ we have $\bar{t} D_{\bar{t}}(G)=X_{\Sigma}(G)$. This proves our claim. 
We next follow the Arens-Singer paper in introducing a foliation of the 3-manifold $\Sigma_{0}$ by a one-parameter family of Riemann surfaces $\Lambda_{\theta}$. We then shall prove

Theorem 2.1. Fix $f$ in $C^{\infty}(\Sigma)$. Then $X f=0$ on $\Sigma$ if and only if the restriction of $f$ to $\Lambda_{\theta}$ is holomorphic on $\Lambda_{\theta}$ for each $\theta$.

We denote by $H^{+}$the right half-plane: $\operatorname{Re} \zeta>0$. For each $\theta \in[0,2 \pi]$ we put $\chi_{\theta}(\zeta)=(\exp (-\zeta), \exp i \theta \exp (-\alpha \zeta))$, where $\zeta$ is in the closed right half-plane.

Definition. Fix $\theta . \Lambda_{\theta}$ is the image in $C^{2}$ of $H^{+}$under the map $\chi_{\theta}$.

Since $|\exp i \theta \exp (-\alpha \zeta)|=|\exp (-\zeta)|^{\alpha}, \Lambda_{\theta}$ is a subset of $\Sigma$. The map $\chi_{\theta}$ is oneone from $H^{+}$to $\Lambda_{\theta}$. We use this map to give $\Lambda_{\theta}$ the structure of a Riemann surface. We verify that for $\theta$ and $\theta^{\prime}$ distinct points in $[0,2 \pi]$, the sets $\Lambda_{\theta}$ and $\Lambda_{\theta^{\prime}}$ are disjoint.

For a function $g$ defined on $\Lambda_{\theta}$, we say that " $g$ is holomorphic on $\Lambda_{\theta}$ " if the composition $\zeta \rightarrow g\left(\chi_{\theta}(\zeta)\right)$ is holomorphic on $H^{+}$.

Now fix $f$ in $C(\Sigma)$ with $X f=0$ on $\Sigma$. Fix $\theta_{0}$. We must show that $f$, restricted to $\Lambda_{\theta_{0}}$, is holomorphic on $\Lambda_{0}$.

Let $\left(z_{0}, w_{0}\right)$ be a point on $\Lambda_{\theta_{0}}$. We fix a single-valued branch of the function $z^{\alpha}$ in a neighborhood $\left|z-z_{0}\right|<\delta$ and fix $\epsilon>0$. Put $U_{\epsilon}=\left\{\left(z, z^{\alpha} \exp i \theta\right)|| z-z_{0} \mid<\right.$ $\left.\delta,\left|\theta-\theta_{0}\right|<\epsilon\right\}$. Let $D$ be the disk $\left\{\left(z, z^{\alpha} \exp i \theta_{0}\right),\left|z-z_{0}\right|<\delta\right\}$, so $D \subset \Lambda_{0}$.

Choose a test function $\phi$ in $C_{0}^{\infty}(D)$, and extend $\phi$ to a smooth function $\tilde{\phi}_{\epsilon}$ in $C_{0}^{\infty}\left(U_{\epsilon}\right)$.

Since $X f=0$, by hypothesis, $X_{\Sigma} f=0$ on $U$ (where we suppress the subscript $\epsilon$ ). So by Claim $1, \bar{t} D_{\bar{t}}(f)=0$ as a distribution on $U$. Therefore, $D_{\bar{t}}(f)=0$ as a distribution on $U$. So $\left\langle D_{\bar{t}}(f), \tilde{\phi}_{\epsilon}\right\rangle=-\int_{U} f D_{\bar{t}} \tilde{\phi}_{\epsilon}=-\int_{D} d t \wedge d \bar{t} \int_{\theta_{0}-\epsilon}^{\theta_{0}+\epsilon} f\left(t, \exp i \theta t^{\alpha}\right) D_{\bar{t}} \tilde{\phi}_{\epsilon} d \theta$.

Since $D_{\bar{t}}(f)=0$ on $U$, we get for each $\epsilon>0: 0=\int_{D} d t d \bar{t} \epsilon^{-1} \int_{\theta_{0}-\epsilon}^{\theta_{0}+\epsilon} f D_{\bar{t}} \tilde{\phi}_{\epsilon} d \theta$, where the integrand of the inner integral is evaluated at $\left(t, \exp i \theta t^{\alpha}\right)$. As $\epsilon$ approaches zero, we get in the limit

$$
0=\int_{D} f\left(t, \exp i \theta_{0} t^{\alpha}\right) D_{\bar{t}} \phi\left(t, \exp i \theta_{0} t^{\alpha}\right) d t \wedge d \bar{t}=\left\langle f, D_{\bar{t}} \phi\right\rangle .
$$

So $D_{\bar{t}}(f)=0$, since this holds for every test function $\phi$ on $D$. Since $D$ is an arbitrary small disk on $\Lambda_{\theta_{0}}, D_{\bar{t}}(f)=0$ as a distribution on $\Lambda_{0}$. By Weyl's Lemma, then, $f$, restricted to $\Lambda_{\theta_{0}}$, is holomorphic on $\Lambda_{\theta_{0}}$.

Conversely, fix $f \in C\left(\Sigma_{0}\right)$ such that $f$ restricted to $\Lambda_{\theta}$ is holomorphic on $\Lambda_{\theta}$ for each $\theta$. We must show that $X f=0$ on $U$, where we write $X$ for $X_{\Sigma}$.

Fix $\left(z_{0}, w_{0}\right)$ in $\Sigma_{0}$. Thus $w_{0}=z_{0}^{\alpha} \exp i \theta_{0}$, for some $\theta_{0}$. We choose a branch of the function $z^{\alpha}$ and also fix $b>0$, and form the set

$$
U_{b}=\left\{\left(z, z^{\alpha} \exp i \theta\right)|| z-z_{0}|<b,| \theta-\theta_{0} \mid<b\right\} .
$$

We claim that $D_{\bar{t}} f=0$ on $U_{b}$. Choose a test function $\phi$ on $U_{b}$. We define

$$
I=\int_{U_{b}} f D_{\bar{t}} \phi d t d \bar{t} d \theta=\left\langle D_{\bar{t}} f, \phi\right\rangle .
$$

We choose a sequence of smooth functions $\left\{f_{n}\right\}$ on $U_{b}$ such that for each $n$ the restriction of $f_{n}$ to $\Lambda_{\theta}$ is holomorphic on $\Lambda_{\theta}$ for each $\theta$ in $\left[\theta_{0}-b, \theta_{0}+b\right]$ and $f_{n}$ converges to $f$ uniformly on $U_{b}$ as $n \rightarrow \infty$ We fix $n$. Put

$$
I_{n}=\int_{U_{b}} f_{n} D_{\bar{t}} \phi d t d \bar{t} d \theta=-\int_{U_{b}} D_{\bar{t}} f_{n} \phi d_{\bar{t}} d \theta
$$


Since $f_{n}$ is holomorphic on $\Lambda_{\theta}$ for each $\theta, I_{n}$ vanishes. Letting $n \rightarrow \infty$, we have $I_{n} \rightarrow I$. So $I=0$.

This holds for all test functions $\phi$ on $U_{b}$. So $D_{\bar{t}} f=0$ as a distribution on $U_{b}$. Since $X f=\bar{t} D_{\bar{t}} f$ on $U_{b}$, then $X f=0$ on $U_{b}$. Since $\left(z_{0}, w_{0}\right)$ is an arbitrary point on $\Sigma_{0}, X f=0$ on $\Sigma_{0}$.

Theorem 2.1 is proved. We now proceed to the proof of Theorem 1.1.

Proof. For $f$ in $C\left(T^{2}\right)$, we put $\|f\|=\max |f|$, taken over $T^{2}$. We define $\mathcal{A}=\{f \in$ $\left.C\left(T^{2}\right)\right\}$ such that $f$ has a continuous extension to $\Sigma$, denoted $F$, with $X F=0$ on $\Sigma_{0}$, in the sense of distributions. Fix $f$ in $\mathcal{A}$. By Theorem 2.1, then, $F$, restricted to $\Lambda_{\theta}$, is holomorphic on $\Lambda_{\theta}$ for each $\theta$; i.e., $F\left(\chi_{\theta}\right)$ is holomorphic on $H^{+}$, where $\chi_{\theta}$ was defined above. Also, since $F$ is continuous on the compact set $\Sigma, F\left(\chi_{\theta}\right)$ is bounded on $H^{+}$. Finally, for $\zeta=i t, t$ real, where $\chi_{\theta}(\zeta)=(\exp i t, \exp i \theta \exp -i \alpha t) \in T^{2}$, $\left|F\left(\chi_{\theta}(\zeta)\right)\right| \leq\|f\|$.

By the Phragmén-Lindelöf theorem, then, $\left|F\left(\chi_{\theta}(\zeta)\right)\right| \leq|| f \|$ for all $\zeta \in H^{+}$, so $|F| \leq\|f\|$ on $\Sigma$. Thus the functions in $\mathcal{A}$, viewed on $\Sigma$, satisfy the maximum principle relative to $T^{2}$. We note that $\mathcal{A}$ is a linear space of functions.

Claim 2. $\mathcal{A}$ is closed under uniform convergence on $T^{2}$.

Proof of Claim 2. Let $\left\{f_{n}\right\}$ be a sequence of functions in $\mathcal{A}$ which converges uniformly on $T^{2}$ to a function $f$. Fix $\left(z_{0}, w_{0}\right) \in \Sigma$. For each of the indices $n, m$, we have

$$
\left|F_{n}\left(z_{0}, w_{0}\right)-F_{m}\left(z_{0}, w_{0}\right)\right| \leq\left\|f_{n}-f_{m}\right\|,
$$

since $f_{n}-f_{m} \in \mathcal{A}$. Hence as $n, m$ tend to $\infty, F_{n}$ converges, uniformly on $\Sigma$, to some continuous function $F$, and $F=f$ on $T^{2}$. Furthermore, for each of the Riemann surfaces $\Lambda_{\theta}$, each $F_{n}$ is holomorphic. Hence $F$ is holomorphic on $\Lambda_{\theta}$. By Theorem 2.1, then, $F$ satisfies $X F=0$ on $\Sigma_{0}$. So $f$ again belongs to $\mathcal{A}$. This was the claim.

Claim 3. $\mathcal{A}$ is an algebra of functions on $T^{2}$.

Proof of Claim 3. Let $f, g \in \mathcal{A}$, and let $F, G$ be their corresponding extensions to $\Sigma$. Since $F$ and $G$ are continuous on $\Sigma$, so is $F G$, and since $F$ and $G$ are each holomorphic on $\Lambda_{\theta}$ for every $\theta$, so is $F G$. Hence by Theorem 2.1, $X(F G)=0$ on $\Sigma_{0}$. Also $F G$ is a continuous extension of $f g$ from $T^{2}$ to $\Sigma$. So $f g$ lies in $\mathcal{A}$. Claim 3 is proved.

Claim 4. $\mathcal{A}$ contains $A_{\alpha}$.

Proof of Claim 4. By Fejér's theorem, $A_{\alpha}$ is the closed span in $C\left(T^{2}\right)$ of the set of functions $\phi_{n, m}=\exp i n \theta \exp i m \phi, n+m \alpha>0$. Fix $n, m$ with $n+m \alpha>0$. We claim that $\phi_{n, m}$ lies in $\mathcal{A}$. With $z, w$ the complex coordinates in $C^{2}$, we consider the extension $z^{n} w^{m}$ of $\phi_{n, m}$ to $\Sigma$. The continuity is clear except at the origin. For $z, w \epsilon \Sigma_{0}$,

$$
\left|z^{n} w^{m}\right|=|z|^{n}|z|^{m \alpha}=|z|^{n+m \alpha} .
$$

As $(z, w) \rightarrow(0,0)$, this tends to 0 . So $z^{n} w^{m}$ provides a continuous extension of $\phi_{n, m}$ to $\Sigma$. Further, $X\left(z^{n} w^{m}\right)=0$ on $\Sigma_{0}$, since $z^{n} w^{m}$ extends to be holomorphic in a neighborhood of $\Sigma_{0}$ in $C^{2}$. So $z^{n} w^{m}$ provides the desired extension of $\phi_{n, m}$, and so $\phi_{n, m} \in \mathcal{A}$. Since $A_{\alpha}$ is the closed span of the $\phi_{n, m}$ in $C\left(T^{2}\right), A_{\alpha}$ is contained in $\mathcal{A}$. Claim 4 is proved. 
By Claims 1 and 2, we know that $\mathcal{A}$ is closed under uniform convergence on $T^{2}$ and is an algebra of functions on $T^{2}$. By Claim 3, $\mathcal{A}$ contains $A_{\alpha}$. Theorem 2.3 in Chapter 7 of T.W. Gamelin's book 2 gives that $A_{\alpha}$ is a maximal subalgebra of $C\left(T^{2}\right)$; i.e., no closed subalgebra of $C\left(T^{2}\right)$ lies properly between $A_{\alpha}$ and $C\left(T^{2}\right)$. So $A_{\alpha}=\mathcal{A}$. Theorem 1.1 is proved.

We proceed to the proof of Theorem 1.2.

Proof. We use the earlier notation.

Claim. There exist integers $p_{j}, q_{j} \in Z^{+}, j=1,2, \ldots$, such that

(1) $-p_{j}+\alpha q_{j}>0$ for all $j$, and

(2) $-p_{j}+\alpha q_{j} \rightarrow 0$, as $j \rightarrow \infty$.

Proof of Claim. A classical fact from the theory of continued fractions (see Hardy and Wright [3], Chapter X) gives the existence of a sequence of rational numbers $\frac{p_{j}}{q_{j}}$ such that

(3) $\left|\alpha-\frac{p_{j}}{q_{j}}\right|<\frac{1}{q_{j}^{2}}, j=1,2, \ldots$ such that $p_{j}$ and $q_{j}$ are positive integers tending to $\infty$ as $j \rightarrow \infty$, and $\frac{p_{j}}{q_{j}}<\alpha$ for each $j$.

Thus for each $j$, we have $\alpha=\frac{p_{j}}{q j}+\delta_{j}$, with $0<\delta_{j}<\frac{1}{q_{j}^{2}}$. It follows that we have $-p_{j}+\alpha q_{j}=q_{j} \delta_{j}$. In view of the bound on $\delta_{j}$, then, we have (1) and (2). So the Claim is proved.

Let $\left\{\epsilon_{n}\right\}$ be a sequence of real numbers tending to 0 . Fix a point $\left(z_{0}, z_{0}^{\alpha}\right)$ in $\Sigma$. We now define a sequence of bounded linear functionals $L_{n}$ on $A_{\alpha}$, as follows:

For $f$ in $A_{\alpha}$, and $F$ denoting the extension of $f$ to $\Sigma$, we put

$$
L_{n} f=\left(\epsilon_{n}\right)^{-1}\left(F\left(z_{0}, \exp i \epsilon_{n} z_{0}^{\alpha}\right)-F\left(z_{0}, z_{0}^{\alpha}\right)\right) .
$$

Let $p_{j}, q_{j}$ be as in the Claim. Define $f_{j}=\exp -i p_{j} \theta \exp i q_{j} \theta$ on $T^{2}$. Since $-p_{j}+\alpha q_{j}>0$, by (1), $f_{j} \in A_{\alpha}$. Further, for $(z, w) \in \Sigma, F_{j}(z, w)=z^{-p_{j}} w^{q_{j}}$. So

$$
\begin{aligned}
L_{n} f_{j}=\left(\epsilon_{n}^{-1}\right)\left(z_{0}^{-p_{j}}\left(\exp i \epsilon_{n} z_{0}^{\alpha}\right)^{q_{j}}-z_{0}^{-p_{j}}\left(z_{0}^{\alpha}\right)^{q_{j}}\right) & =\left(\epsilon_{n}^{-1}\right)\left(z_{0}^{-p_{j}} z_{0}^{\alpha q_{j}}\right)\left(\exp i \epsilon_{n} q_{j}-1\right) \\
& =\left(z_{0}^{-p_{j}+\alpha q_{j}}\right)\left(\frac{\exp i \epsilon_{n} q_{j}-1}{\epsilon_{n}}\right)
\end{aligned}
$$

We now take $j=n$ and take absolute values. We get

$$
\left|L_{n}\left(f_{n}\right)\right|=\left(\left|z_{0}\right|^{-p_{n}+\alpha q_{n}}\right)\left(\epsilon_{n}^{-1}\right)\left|\left(\exp i \epsilon_{n} q_{n}-1\right)\right| .
$$

We next take $\epsilon_{n}=\frac{\pi}{q_{n}}$. This gives $\left|L_{n}\left(f_{n}\right)\right|=\left|z_{0}\right|^{-p_{n}+\alpha q_{n}}\left(\frac{2}{\pi}\right) q_{n}$. Since $-p_{n}+$ $\alpha q_{n} \rightarrow 0$ and $q_{n} \rightarrow \infty$ as $n \rightarrow \infty,\left|L_{n}\left(f_{n}\right)\right| \rightarrow \infty$ as $n$ approaches $\infty$. Also, $\left\|f_{n}\right\|=1$ for each $n$. So the norm $\left\|L_{n}\right\|$, as a functional on $A_{\alpha}$, becomes unbounded as $n$ grows.

Next, we fix $f \in A_{\alpha}$ and the point $x_{0}=\left(z_{0}, z_{0}^{\alpha}\right)$. $F$ denotes the extension of $f$ to $\Sigma$. We put $\Psi(\theta)=F\left(z_{0}, \exp i \theta z_{0}^{\alpha}\right),-\pi \leq \theta \leq \pi$. Then

$$
L_{n}(f)=\left(\epsilon_{n}^{-1}\right)\left(F\left(\left(z_{0}, \exp i \epsilon_{n} z_{0}^{\alpha}\right)\right)-F\left(z_{0}, z_{0}^{\alpha}\right)\right)=\left(\epsilon_{n}^{-1}\right)\left(\Psi\left(\epsilon_{n}\right)-\Psi(0)\right) .
$$

Suppose now that $F$ is differentiable on $\Sigma$ at $x_{0}$. Then $\Psi$ is differentiable at $\theta=0$. Hence by the preceding equality, the sequence $\left\{L_{n}(f)\right\}$ converges as $n$ approaches $\infty$. Since $L_{n}$ converges pointwise on the Banach space $A_{\alpha}$, the uniform boundedness theorem yields that the sequence $\left\{L_{n}\right\}$ is bounded. This contradicts our earlier result. So for some $f$ in $A_{\alpha}, F$ fails to be differentiable at the given point. Theorem 1.2 is proved. 


\section{ACKNOWLEDGMENT}

The author is grateful to J.J. Kohn for having remarked on some occasion, "People who study function algebras should look at the solution space of first order linear differential operators." The above is one example where this paid off.

\section{REFERENCES}

[1] R. Arens and I. Singer, Generalized analytic functions, Trans. Amer. Math. Soc. 81 (1956), 379-393. MR0078657 (17:1226e)

[2] T. W. Gamelin, Uniform Algebras, Prentice Hall, Inc., 1969. MR0410387 (53:14137)

[3] G. H. Hardy and E. M. Wright, An Introduction to the Theory of Numbers, 2nd edition, Oxford, 1945. MR0067125 (16:673c)

[4] H. Helson and D. Lowdenslager, Prediction theory and Fourier series in several variables, Acta Math. 99 (1958), 165-202. MR0097688(20:4155)

[5] K. Hoffman and I. M. Singer, Maximal subalgebras of $C(\Gamma)$, Amer. Jour. of Math. 79 (1957), 295-305. MR0085478(19:46e)

Department of Mathematics, Brown University, 151 Thayer Street, Providence, RHode IsLAND 02912

E-mail address: wermer@math.brown.edu 